\title{
Two-Dimensional Natural Convection Heat Transfer from a Heated Plate Immersed in a Thermally Stratified Medium: Numerical and Experimental Study
}

\author{
Naseem Ali \\ PhD student, Department of Mechanical \& Materials Engineering \\ Portland State University \\ USA
}

\author{
Ihsan Y. Hussain \\ Professor, Department of Mechanical Engineering \\ Baghdad University \\ Iraq
}

\begin{abstract}
The effect of thermal stratification in stable stationary ambient fluid on free convective flow of a viscous incompressible fluid along a plane wall is numerically and experimentally investigated in the present work. The governing equations of continuity, momentum and energy are solved numerically using finite difference method with Alternating Direct Implicit scheme. The velocity, temperature distributions and the Nusselt number are discussed numerically for various values of physical parameters and presented through graphs. Experiments are carried out in an apparatus, consisting of a square Perspex aquarium and heated plate. The measurements are conducted using 12 channel temperature recorders. The temperature profiles and Nusselt number for stratified and unstratified media are compared with corresponding numerical solution and good agreement is obtained. The results show that the thickness of the thermal boundary layer is approximately independent of downstream coordinates.
\end{abstract}

\section{Keywords}

Thermal stratification, free convection, Alternating Direct Implicit

\section{INTRODUCTION}

In natural convection, as in other convective processes, a consideration of fluid flow is necessary in the study of the energy and mass transfer mechanisms. A study of convection further necessitates a consideration of the coupling between the fluid flow and the mechanisms underlying conduction. This is due to the fact says that the heat transported due to a moving fluid element would eventually be transferred to its neighboring elements through conduction. Moreover, in region close to a surface which is at temperature different from that of the ambient medium there is no relative motion between the surface and the fluid, and the transfer of energy is predominantly through conduction. The principle of natural convection can be classified depending on the nature of the ambient medium outside the boundary layer. In the most various processes the ambient medium considered as isothermal and uniform density whereas there are several states of interest in which the fluid density is varying with height and /or time; this phenomenon is known as stratification. In the absence of diffusion a stably stratified medium continues to exist, whereas an unstable stratification gives rise to convective flow which would lead to a disruption of the stratification. A medium would be stably stratified when a lighter fluid lies over a heavier one. Therefore, the condition for existence of a stable stratification is that the density must decrease vertically. For the circumstance, where the density may be taken as a function of temperature alone, this condition requires that the fluid temperature increases vertically. The thermal stratification can be classified depending on the temperature gradient into linear thermal stratification when the gradient temperature is constant and non-linear thermal stratification" when the gradient is varying. The time is considered as effective factor on the thermal stratification medium which acts to destratify the medium and lead it to fully mixed (unstratified medium). Convective heat transfer in thermally stratified ambient fluid occurs in many industrial applications and is an important aspect in the study of heat transfer. If stratification occurs, the fluid temperature is function of distance and convection in such environment exists in lakes, oceans, nuclear reactors. The problem had been investigated by many researcher analytically and numerically, see for example [1, 2, 3, 5, 7, 8, 9, 10, 11, 12, 13, 15]. Experimental works also had been reported; see [14]. Theoretical and experimental work had been investigate by [4]. The present work investigates the problem numerically with wide range of stratification parameter for different kinds of fluids (air, water, and oil), different Grashof number and different inclination angle. To support the numerical solution the problem was investigated experimentally also.

\section{MATHEMATICAL MODEL}

To get the final form for governing equations, many assumptions are taken in consideration. The following assumptions are used in the modeling. Consider the two dimensional thermal boundary layer flow of natural convection heat transfer of an incompressible fluid along a plane wall immersed in a stable thermally stratified fluid. The physical properties are constant except the density in the buoyancy term of momentum equation, which varies according to Boussinesq approximation. Negligible viscous dissipation in the energy equation due to the small velocities of the natural convection. Negligible internal heat generation and radiation are assumed. The following continuity, momentum and energy equations in non-dimensional form for laminar flow adjacent to a plane wall are obtained; 


$$
\begin{gathered}
\frac{\partial U}{\partial X}+\frac{\partial V}{\partial Y}=0 \\
\frac{\partial U}{\partial t^{*}}+U \frac{\partial U}{\partial X}+V \frac{\partial U}{\partial Y}=\frac{1}{\sqrt{G r}}\left(\frac{\partial^{2} U}{\partial X^{2}}+\frac{\partial^{2} U}{\partial Y^{2}}+\theta\right) \\
\frac{\partial V}{\partial t^{*}}+U \frac{\partial V}{\partial X}+V \frac{\partial V}{\partial Y}=\frac{1}{\sqrt{G r}}\left(\frac{\partial^{2} V}{\partial X^{2}}+\frac{\partial^{2} V}{\partial Y^{2}}+\theta \tan \psi\right)
\end{gathered}
$$

where $X=x / L, Y=y / L, U=u / u_{c}, V=v / u_{c}$, $t^{*}=t u_{c} / L, \operatorname{Pr}=\mu c_{p} / k, u_{c}=\sqrt{g \cos \psi \beta \Delta T_{0} L}, \theta=\frac{T-T_{\infty, 0}}{T_{w}-T_{\infty, 0}}$, $G r=\frac{g \beta \cos \psi L^{3} \Delta T_{0}}{\nu^{3}}, \beta=-\frac{1}{\rho}\left(\frac{\partial \rho}{\partial T}\right)_{p}$ and $S=\frac{1}{\Delta T_{0}}\left(\frac{d T_{\infty, 0}}{d X}\right)$.

The nomenclature is defined as Grashof number $(G r)$, gravitational acceleration $(g)$, characteristic length of the plane wall $(L)$, Nusselt number $(N u)$, Prandtl number $(P r)$, thermal stratification parameter $(S)$, temperature $(T)$, time $(t)$, non-dimensional time $\left(t^{*}\right)$, velocity in $\mathrm{x}$-direction $(u)$, characteristic velocity $\left(u_{c}\right)$, non-dimensional velocity in $\mathrm{X}$ direction $(U)$, velocity in y-direction $(v)$, non-dimensional velocity in $Y$ direction $(V)$, non-dimensional downstream coordinate $(X)$, downstream coordinate $(x)$, non-dimensional horizontal space coordinate $(Y)$, horizontal space coordinate $(y)$, kinematic viscosity $(\nu)$, density $(\rho)$, angle of inclination $(\psi)$, non-dimensional temperature $(\theta)$, thermal diffusivity $(\alpha)$, volumetric coefficient of thermal expansion $(\beta)$, location away from the wall outside the boundary layer $(\infty)$, location away from the wall at $x=0(\infty, 0)$, location away from the wall at any $X(\infty, X)$, and wall $(w)$.

The initial condition can be written in non-dimensional form as follows:

$$
\begin{gathered}
U=0, V=0, \frac{\partial \theta}{\partial Y}=\frac{q_{w}^{\prime \prime} L}{k \Delta_{0}} \text { for } Y=0 \text { for all } X \\
U=0, V=0, \theta=0 \text { for } Y=\infty \text { for all } X \\
U=0, V=0, \theta=0 \text { for } Y=\infty \text { and } X=0
\end{gathered}
$$

Natural convection heat transfer on a surface depends on the geometry of the surface as well as its orientation. It also depends on the variation of temperature on the surface and the thermophysical properties of the fluid involved. The Nusselt number is one of important dimensionless numbers because it represents the enhancement of heat transfer through a fluid layer as a result of convection relative to conduction across the same fluid layer. The Nusselt numbers have the functional form for natural convection in a stratified media $N u_{x}=f(X, G r, P r, S)$. The local rate of heat transfer in term of the local Nusselt number at the plate is given by,

$$
N u_{x}=-\frac{x}{\theta_{w}}\left(\frac{\partial \theta}{\partial Y}\right)_{Y=0} .
$$

The thickness of the thermal boundary layer was defined as the distance at which, (Tanny and Cohen 1998);

$$
\frac{T-T_{\infty, 0}}{T_{w}-T_{\infty, 0}}=0.5
$$

In the present work the new definition of the thickness of thermal boundary layer will be the distance at which the temperature profile equal to half wall temperature profile.

$$
\frac{\theta}{\theta_{w}}=0.5
$$

\section{NUMERICAL SOLUTION}

Finite Difference Method is considered as efficient technique to solve the thermal problems; therefore it has been used in the present study. The momentum and energy equations are solved by Alternating Direction Implicit scheme (ADI). Numerical results were first obtained to check for grid dependency. The results showed that no considerable difference in the results of suggested grid size after (51x51) and showed that no considerable different in the results of suggested transverse distance after (0.5). Therefore in the present study the grid size of $(51 \times 51)$ and transverse distance of (0.5) was used. The convergence of the solution to the steady state result for large time was obtained with a convergence criterion of $\left(1 \times 10^{-4}\right)$. This criterion was chosen after varying it over a wide range so that the steady state results were essentially independent of the chosen value. The mathematical model was solved by computer program which was written by the Visual basic language to solve the momentum and energy equations and to calculate Nusselt number. The Tridiagonal system of equation was used to solve the matrix of dependent variables.

In ADI, the discretized time step is divided into two periods as, the first time period $(t=0: n+0.5)$ and the second time period $(t=n+0.5: n+1)$. The momentum equation is discretized in $X$-direction as,

$$
\begin{aligned}
& \frac{2}{\Delta t^{*}}\left(U_{i, j}^{n+0.5}-U_{i, j}^{n}\right)+\frac{U_{i, j}^{n}}{2 \Delta X}\left(U_{i+1, j}^{n+0.5}-U_{i-1, j}^{n+0.5}\right)+\frac{V_{i, j}^{n}}{2 \Delta Y}\left(U_{i, j+1}^{n}-\right. \\
& \left.U_{i, j-1}^{n}\right)=\frac{1}{\sqrt{G r}}\left[\frac{1}{(\Delta X)^{2}}\left(U_{i+1, j}^{n+0.5}-2 U_{i, j}^{n+0.5}+U_{i-1, j}^{n+0.5}\right)+\right. \\
& \left.\frac{1}{(\Delta Y)^{2}}\left(U_{i, j+1}^{n}-2 U_{i, j}^{n}+U_{i, j-1}^{n}\right)\right]+\theta_{i, j}^{n} . \\
& \frac{2}{\Delta t^{*}}\left(U_{i, j}^{n+1}-U_{i, j}^{n+0.5}\right)+\frac{U_{i, j}^{n}}{2 \Delta X}\left(U_{i+1, j}^{n+0.5}-U_{i-1, j}^{n+0.5}\right)+ \\
& \frac{V_{i, j}^{n+0.5}}{2 \Delta Y}\left(U_{i, j+1}^{n+1}-U_{i, j-1}^{n+1}\right)=\frac{1}{\sqrt{G r}}\left[\frac { 1 } { ( \Delta X ) ^ { 2 } } \left(U_{i+1, j}^{n+0.5}-2 U_{i, j}^{n+0.5}+\right.\right. \\
& \left.\left.U_{i-1, j}^{n+0.5}\right)+\frac{1}{(\Delta Y)^{2}}\left(U_{i, j+1}^{n+1}-2 U_{i, j}^{n+1}+U_{i, j-1}^{n+1}\right)\right]+\theta_{i, j}^{n} .
\end{aligned}
$$

Thus, the numerical analysis to momentum equation in $Y$-direction is determined as,

$$
\begin{aligned}
& \frac{2}{\Delta t^{*}}\left(V_{i, j}^{n+0.5}-V_{i, j}^{n}\right)+\frac{U_{i, j}^{n}}{2 \Delta X}\left(V_{i+1, j}^{n+0.5}-V_{i-1, j}^{n+0.5}\right)+\frac{V_{i, j}^{n}}{2 \Delta Y}\left(V_{i, j+1}^{n}-\right. \\
& \left.V_{i, j-1}^{n}\right)=\frac{1}{\sqrt{G r}}\left[\frac{1}{(\Delta X)^{2}}\left(V_{i+1, j}^{n+0.5}-2 V_{i, j}^{n+0.5}+V_{i-1, j}^{n+0.5}\right)+\right. \\
& \left.\frac{1}{(\Delta Y)^{2}}\left(V_{i, j+1}^{n}-2 V_{i, j}^{n}+V_{i, j-1}^{n}\right)\right]+\theta_{i, j}^{n} \tan \psi . \\
& \frac{2}{\Delta t^{*}}\left(V_{i, j}^{n+1}-V_{i, j}^{n+0.5}\right)+\frac{U_{i, j}^{n}}{2 \Delta X}\left(V_{i+1, j}^{n+0.5}-V_{i-1, j}^{n+0.5}\right)+\frac{V_{i, j}^{n}}{2 \Delta Y}\left(V_{i, j}^{n+1}-\right. \\
& \left.V_{i, j-1}^{n+1}\right)=\frac{1}{\sqrt{G r}}\left[\frac{1}{(\Delta X)^{2}}\left(V_{i+1, j}^{n+0.5}-2 V_{i, j}^{n+0.5}+V_{i-1, j}^{n+0.5}\right)+\right. \\
& \left.\frac{1}{(\Delta Y)^{2}}\left(V_{i, j+1}^{n+1}-2 V_{i, j}^{n+1}+V_{i, j-1}^{n+1}\right)\right]+\theta_{i, j}^{n} .
\end{aligned}
$$

Finally, the energy equation is quantified as,

$$
\begin{aligned}
& \frac{2}{\Delta t^{*}}\left(\theta_{i, j}^{n+0.5}-\theta_{i, j}^{n}\right)+\frac{U_{i, j}^{n}}{2 \Delta X}\left(\theta_{i+1, j}^{n+0.5}-\theta_{i-1, j}^{n+0.5}\right)+\frac{V_{i, j}^{n}}{2 \Delta Y}\left(\theta_{i, j+1}^{n}-\right. \\
& \left.\theta_{i, j-1}^{n}\right)=\frac{1}{\operatorname{Pr} \sqrt{G r}}\left[\frac{1}{(\Delta X)^{2}}\left(\theta_{i+1, j}^{n+0.5}-2 \theta_{i, j}^{n+0.5}+\theta_{i-1, j}^{n+0.5}\right)+\right. \\
& \left.\frac{1}{(\Delta Y)^{2}}\left(\theta_{i, j+1}^{n}-2 \theta_{i, j}^{n}+\theta_{i, j-1}^{n}\right)\right]-S U_{i, j}^{n} . \\
& \frac{2}{\Delta t^{*}}\left(\theta_{i, j}^{n+1}-\theta_{i, j}^{n+0.5}\right)+\frac{U_{i, j}^{n}}{2 \Delta X}\left(\theta_{i+1, j}^{n+0.5}-\theta_{i-1, j}^{n+0.5}\right)+\frac{V_{i, j}^{n+1}}{2 \Delta Y}\left(\theta_{i, j+1}^{n+1}-\right. \\
& \left.\theta_{i, j-1}^{n+1}\right)=\frac{1}{\operatorname{Pr} \sqrt{G r}}\left[\frac{1}{(\Delta X)^{2}}\left(\theta_{i+1, j}^{n+0.5}-2 \theta_{i, j}^{n+0.5}+\theta_{i-1, j}^{n+0.5}\right)+\right.
\end{aligned}
$$




$$
\left.\frac{1}{(\Delta Y)^{2}}\left(\theta_{i, j+1}^{n+1}-2 \theta_{i, j}^{n+1}+\theta_{i, j-1}^{n+1}\right)\right]-S U_{i, j}^{n} .
$$

Heat transfer from bodies immersed in a stratified environment can be expressed in terms of Grashof and Prandtl numbers and stratification parameter. The Local Nusselt number $\left(N u_{X}\right)$ at the location $(X)$ for flow over the plane wall can be determined from the following relations,

$$
N u_{X}=-\frac{X}{\theta_{w}}\left[\frac{-25 \theta_{i, 0}+48 \theta_{i, 1}-36 \theta_{i, 2}+16 \theta_{i, 3}-3 \theta_{i, 4}}{12 \Delta Y}\right] .
$$

The average Nusselt number can be calculated by integrating the local Nusselt number over the plane wall length as follows; $N y_{\text {avg }}=\int_{0}^{1} N u_{X} d X$.

\section{EXPERIMENTAL WORK}

The experimental work was conducted in the Heat Transfer Lab. in the Mechanical Engineering department at Baghdad University. It consists of Perspex glass aquarium, which consists of two boxes assembled together. The exterior box was assembled with interior dimensions of $(600 \times 600 \times 600 \mathrm{~mm})$. The interior box was gathered with exterior dimensions of $(500 \times 500 \times 500 \mathrm{~mm})$. The Perspex glass sheets that used for the boxes are with $(10 \mathrm{~mm}$ thickness). Thermal insulator, with (50 mm thickness), was used to fill space between the two boxes, and also the outer surfaces of the exterior box. The heated plate assembly was made of rectangular Aluminum box with overall dimensions of $(200 \times 100 \times 50 \mathrm{~mm})$ over which the surrounding air at a predetermined temperature could circulate. One side of the aluminum box was insulated by thermal insulator. The other side of the aluminum box served as the heating surface unit. The heater coil that used with a $(2 \mathrm{~mm})$ diameter and made from nickel-chrome wire (R). Ceramic beads with diameter $(3 \mathrm{~mm})$ electrically isolate this coil. The cooling system and heater coil were used in test rig to perform the gradient heating of the ambient fluid. The cooling system was consisting of cooling pipes, electrical water pump, water tank and water cooler. The water was used as working fluid in cooling system. The cooling pipes were covered up by commercial Aluminum plate, with (1 mm thickness).The ambient stratification in the aquarium was measured continuously by fixed vertical rake of six Alumel-Chromel (type K) thermocouples. The thermocouples were separated by a uniform vertical distance of a $(30 \mathrm{~mm})$. The temperature profiles across the boundary layer were measured by horizontal rake of six Alumel-Chromel (type K) thermocouples. The horizontal distance between the thermocouple was chosen to cover up the change in temperature profile, first five thermocouples with interval distance of $(5 \mathrm{~mm})$ and the sixth thermocouple with interval distance of $(10 \mathrm{~mm})$. The digital thermometer type (12 Channels Temperature Recorder with SD Card Data Logger) was used.

\section{ERROR ANALYSIS}

The accuracy of obtaining experimental results depends upon two factors. The accuracy of measurements and the design details of test rig. The deviations in accuracy are resulted form. 1.) The uniformities in heat flux on the wall surface. 2.) The alignment of fixing thermocouple in the aquarium. 3.) The straightening of heated plate in vertical position and inclined position. 4.) Heat lost by conduction from side walls. 5.) Linearity of the stratification Parameter. There is no doubt that, the maximum portion of errors in calculations referred essentially to the errors in the measured quantities. Hence, to calculate the error in the obtained results, the procedure of Kline and McClintock [6], is used in this field. Let the result $\mathrm{R}$ be a function of $\mathrm{n}$ independent variables $\left(v_{1}, v_{2} \cdots v_{n}\right)$, For small variations in the variables, this relation can be expressed in linear form as

$$
\delta R=\frac{\partial R}{\partial v_{1}} \delta v_{1}+\frac{\partial R}{\partial v_{2}} \delta v_{2}+\cdots+\frac{\partial R}{\partial v_{n}} \delta v_{n}
$$

Hence, the uncertainty intervals (w) in the result can be given as,

$$
w_{R}=\left[\left(\frac{\partial R}{\partial v_{1}} \times w_{1}\right)^{2}+\left(\frac{\partial R}{\partial v_{2}} \times w_{2}\right)^{2}+\cdots+\left(\frac{\partial R}{\partial v_{n}} \times w_{n}\right)^{n}\right]^{0.5}
$$

Thereafter, the above equation is nondimensionalized as,

$\frac{w_{R}}{R}=\left[\left(\frac{\partial R}{\partial v_{1}} \times \frac{w_{1}}{R}\right)^{2}+\left(\frac{\partial R}{\partial v_{2}} \times \frac{w_{2}}{R}\right)^{2}+\cdots+\left(\frac{\partial R}{\partial v_{n}} \times \frac{w_{n}}{R}\right)^{n}\right]^{0.5}$

Therefore the uncertainty intervals $(w)$ of the result can be given as follows;

$$
\begin{aligned}
& \frac{w_{N u_{X}}}{N u_{X}}=\left[\left(\frac{\partial N u_{X}}{\partial V o} \times \frac{w_{V o}}{N u_{X}}\right)^{2}+\left(\frac{\partial N u_{X}}{\partial I} \times \frac{w_{I}}{N u_{X}}\right)^{2}+\left(\frac{\partial N u_{X}}{\partial X} \times\right.\right. \\
& \left.\left.\frac{w_{X}}{N u_{X}}\right)^{2}+\left(\frac{\partial N u_{X}}{\partial A_{s}} \times \frac{w_{A_{s}}}{N u_{X}}\right)^{2}+\left(\frac{\partial N u_{X}}{\partial \Delta T_{w}} \times \frac{w_{\Delta T_{w}}}{N u_{X}}\right)^{2}\right] .
\end{aligned}
$$

where, $V o, I$ and $A_{s}$ is the voltage, current and surface area, respectively. In this study, the error analysis corresponding average Nusselt number is less than $10 \%$.

\section{NUMERICAL RESULTS}

Theoretical investigation are done for three working fluids, air $(P r$ $=0.7)$, water $(P r=6)$ and oil $(P r=6400)$, three Grashof numbers $\left(1 \times 10^{4}, 1 \times 10^{5}\right.$ and $\left.1 \times 10^{6}\right)$ and three angle $(-30,0$ and 30$)$ for wide range of thermal stratification $(S=0,0.5,1,1.5,2,3,4)$. Figures 1 and 2 show that the temperature profile for the different values of the stratification level at mid high wall plane $(X=0.5)$. The temperature profile does not affect strongly with increasing the stratification parameter. The constant heat flux boundary condition has been marginalized the effect of stratification. A comparative study of figures indicates that the effect of stratification parameter is marginalized with the increase in Prandtl number, as the separateness among the temperature profile reduces. Figure 3 shows that the temperature profile decreases with the increase in Prandtl number. In addition, the reversal of temperature was found to be stronger at high Prandtl numbers and weaker at low numbers. It can be suitably remarked that the increase in Grashof number does not practically vary the effect of stratification factor on temperature profiles. Figure 4 shows that with increase in Grashof number the fluid temperature deceases. This must happen because buoyancy force assists the flow by increasing fluid velocity and hence the heat is convected readily thereby reducing fluid temperature. Figure 5 illustrates the influence of the inclination angle $(\psi)$ on temperature profile for stratified media $(S=2)$, where observed that in addition to the influence of thermal stratification the temperature profile will be less effected by the inclination angle of the wall, this is considerably noted for high levels of thermal stratification, therefore the orientation marginalized the effect of the stratification parameter. Figure 6 shows that the velocity profile does not effected strongly with increasing the stratification parameter and the figure 7 shows that the velocity profile decreases with increasing stratification parameter. The 
limit effect of stratification on velocity profile is a result to the difference between the wall temperature and ambient temperature does not effected strongly by increasing the stratification parameter because the increasing in ambient temperature corresponding to the increasing on the wall temperature. A comparative study of figure indicates that the effect of stratification parameter is marginalized with the increase in Prandtl number, as the separateness among the velocity profile reduces. Figure 8 shows that the velocity profile decreases with the increase in Prandtl number. At high Prandtl numbers there is a small reversal of flow while for low Prandtl numbers the flow reversal is much stronger. It can be suitably remarked that the increase in Grashof number does not practically vary the effect of stratification factor on velocity profiles. Figure 9 shows that with increase in Grashof number the fluid velocity increases. This is because the buoyancy force assists the flow by increasing fluid velocity. Figure 10 shows that the velocity profile decreases with increasing the Grashof number. This phenomenon is clear at high Prandtl number which lowers fluid velocity. Figure 11 illustrates the influence of the inclination angle $(\psi)$ on velocity profile for stratified level $(S=2)$, where observed that in addition to the influence of thermal stratification the velocity profile will be less effected by the inclination angle of the wall, this is considerably noted for high levels of thermal stratification, therefore the orientation marginalized the effect of the stratification parameter. Figures 12 and 13 show that marginalized effect of stratification parameter on the local Nusselt number because the temperature profile does not affected strongly with increasing the stratification parameter. Figure 14 shows that as the Prandtl number increases the Nusselt number first deceases, then increases. The fluids which have small Prandtl number, Nusselt number decreases with increasing the Grashof number, see figure 15.

\section{EXPERIMENTAL RESULTS}

Experimental investigation were done for air as working fluid, with wide range of heat flux $(250,1000,1500,2000$ and 2500 $\mathrm{W} / \mathrm{m} 2)$ and of inclination angle $(-30,-15,0,15$ and 30$)$. The fluid temperature profile has been tested with the transverse coordinates at different downstream coordinates for different heat fluxes. The temperature profiles have been measured for stratified and unstratified fluid. Figure 16 show that the effect of thermal stratification has been marginalized due to heat flux; therefore the thermal stratification does not have clear effect on temperature profile. This state has been proved for all studied range value of heat fluxes. The figure 17 reveals the marginalized effect to inclination angle in stratified media; therefore the temperature profile does affected strongly by change the orientation. This state has been proved for all studied range of heat flux and inclination angle. To examine the effect of the ambient stratification on the heat transfer, the local Nusselt numbers have been measured for stratified and unstratified media. The figure 18 represent comparison between stratified and unstratified media cases for local Nusselt number. The figures reveal that the local Nusselt number increases with downstream coordinates in stratified and unstratified environment, and the heat transfer rate does not affected by thermal stratification therefore the local Nusselt number has been approximately held in stratified and unstratified media. This is a logic result because the local Nusselt number depends on temperature profile which does not affected by the thermal stratification as mention above. Also fluid temperature profile has been tested with the computational domain normal to the wall for different values of downstream coordinates to find the thickness of the thermal boundary layer. Thus, the result shows that the thermal

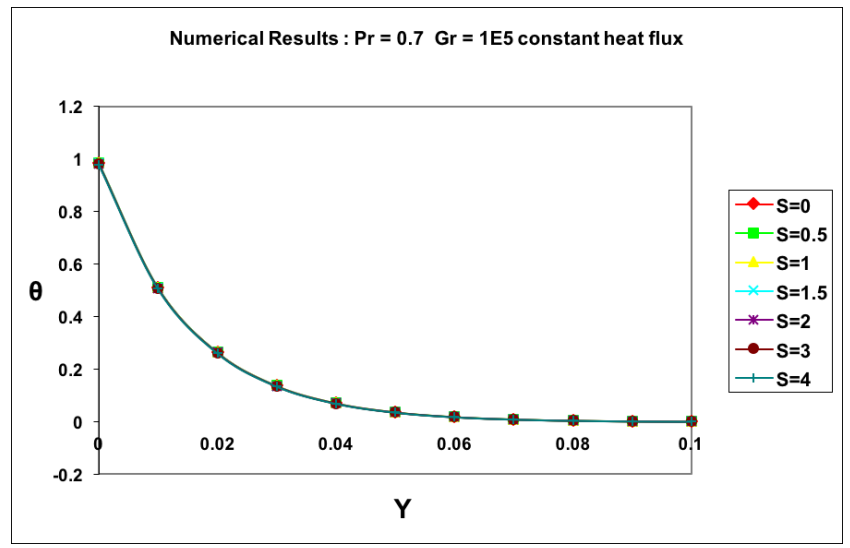

Fig. 1. Temperature profile for $P r=0.7$.

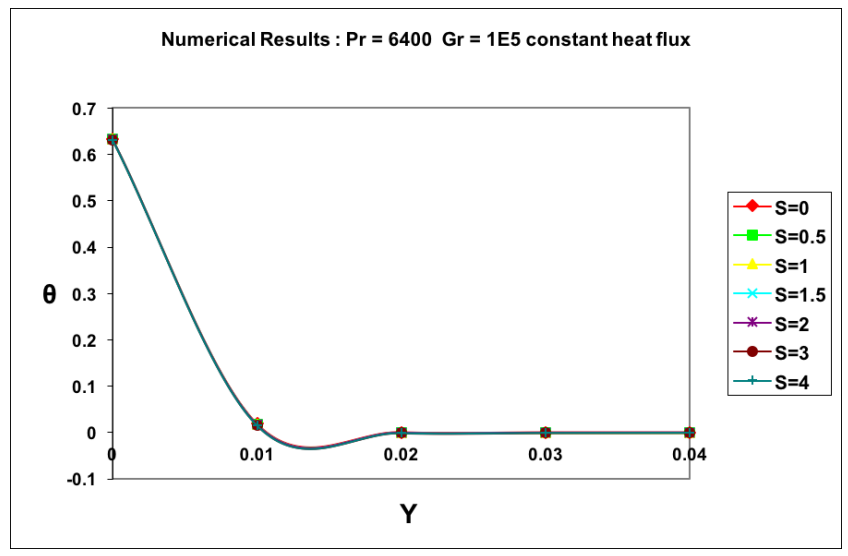

Fig. 2. Temperature profile for $\operatorname{Pr}=6400$.

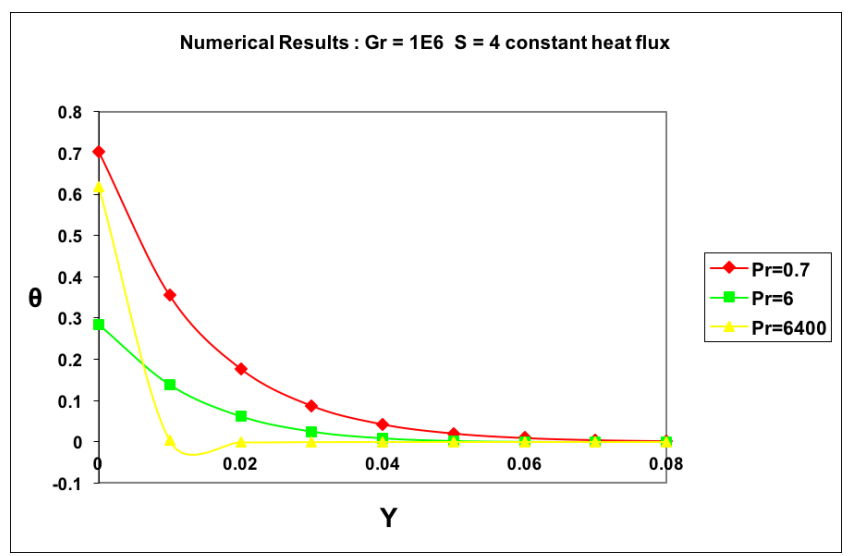

Fig. 3. Temperature profile for $G r=1 \mathrm{E} 6$.

boundary layer is approximately independent on the downstream coordinate. The thickness of the thermal boundary layer was approximately $(2.75 \mathrm{~mm})$. 


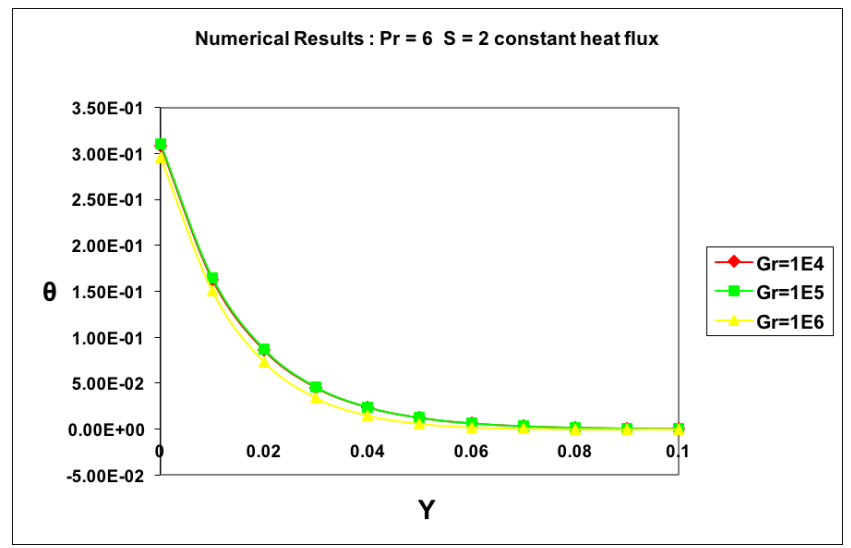

Fig. 4. Temperature profile for $\operatorname{Pr}=6$ and $S=2$.

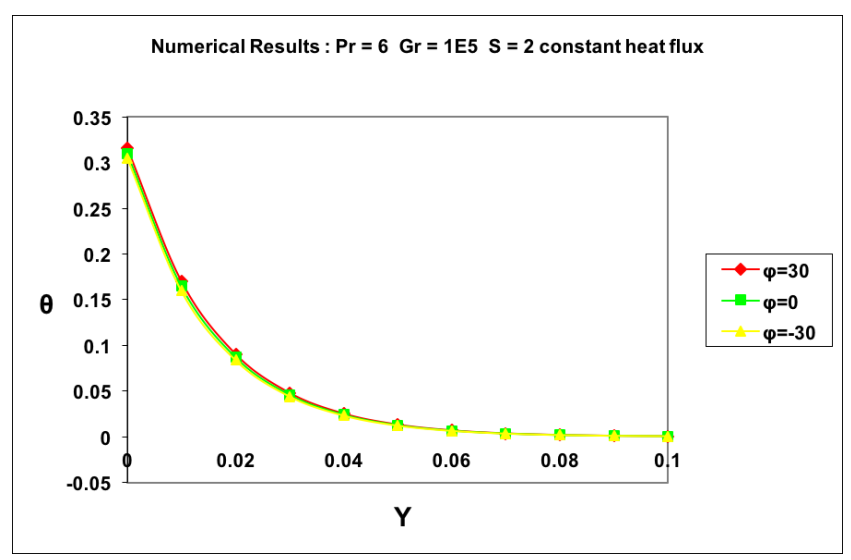

Fig. 5. Temperature Profile for $\operatorname{Pr}=6$ and $S=2$.

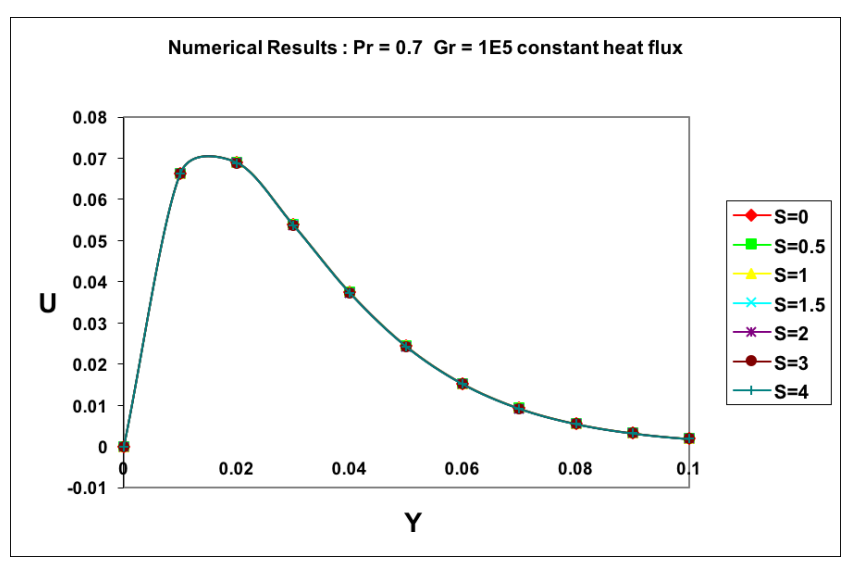

Fig. 6. Velocity profile for $\operatorname{Pr}=0.7$.

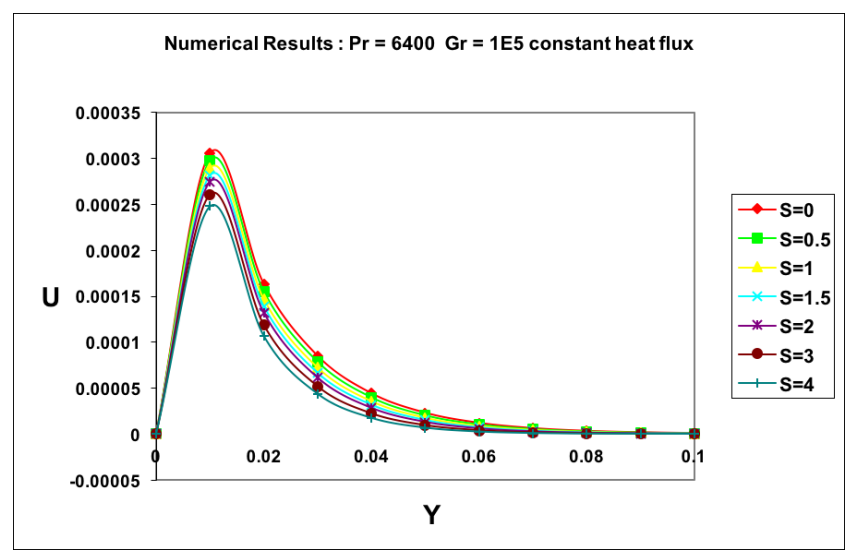

Fig. 7. Velocity profile for $\operatorname{Pr}=6400$.

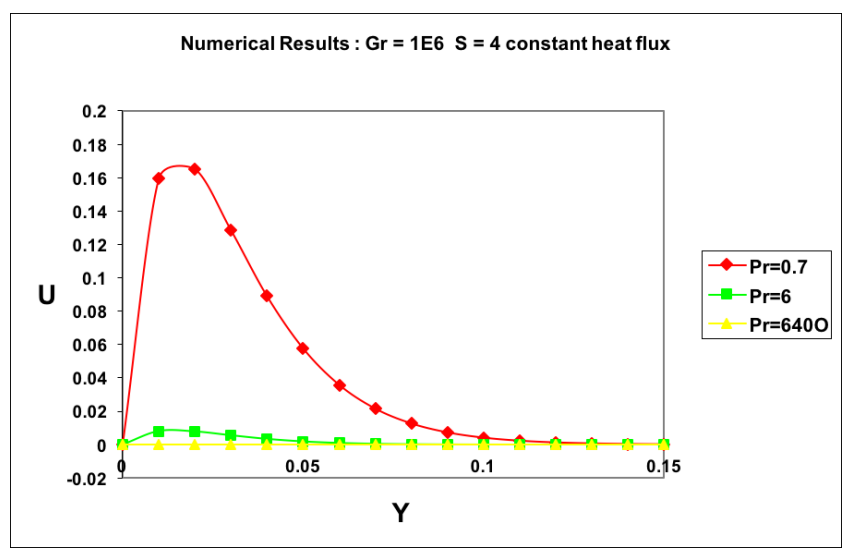

Fig. 8. Velocity profile for $G r=1 \mathrm{E} 6$.

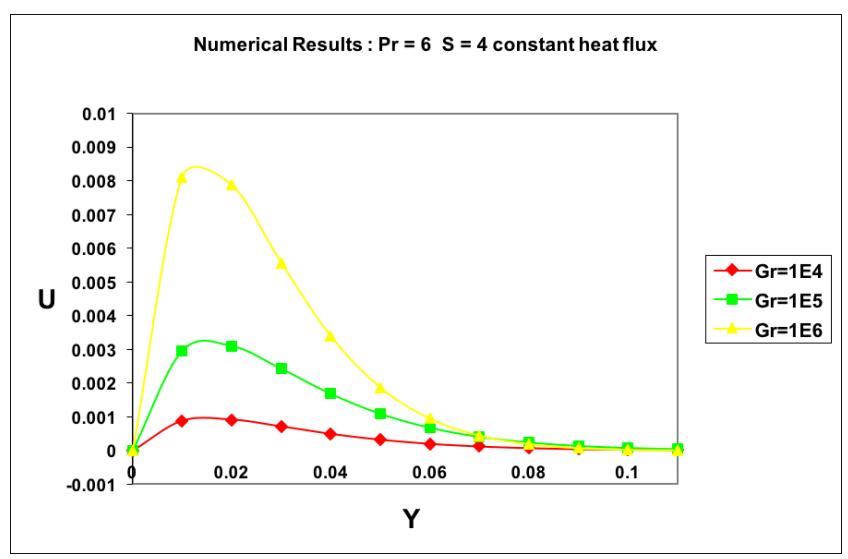

Fig. 9. Velocity profile for $\operatorname{Pr}=6$ and $S=4$. 


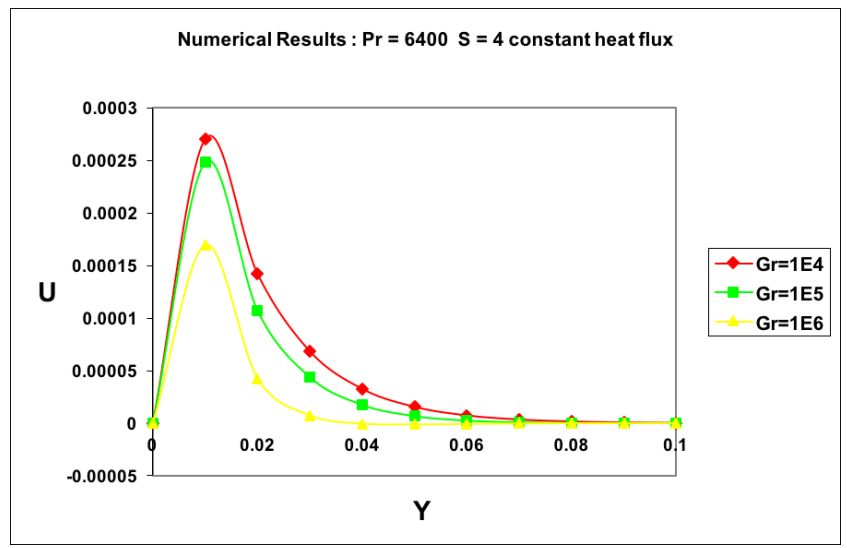

Fig. 10. Velocity profile for $\operatorname{Pr}=6400$ and $S=4$.

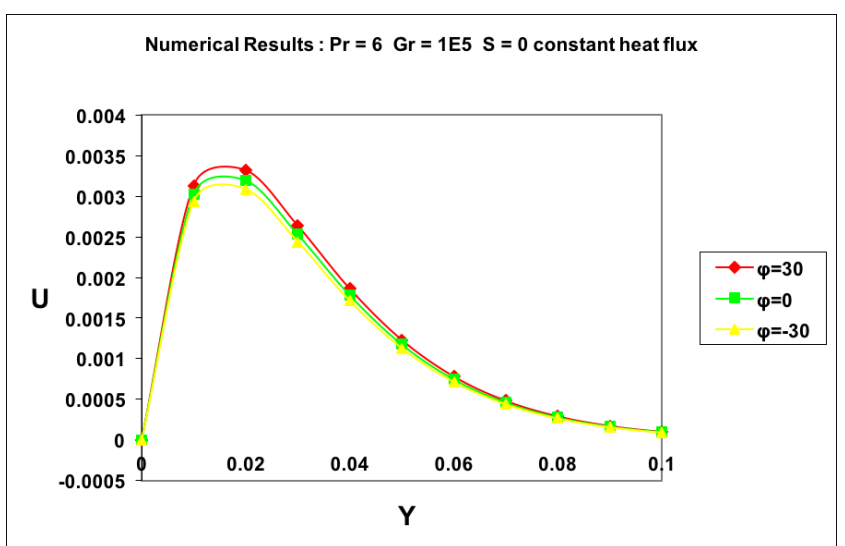

Fig. 11. Velocity profile for $P r=6400$ and $G r=1$ E6.

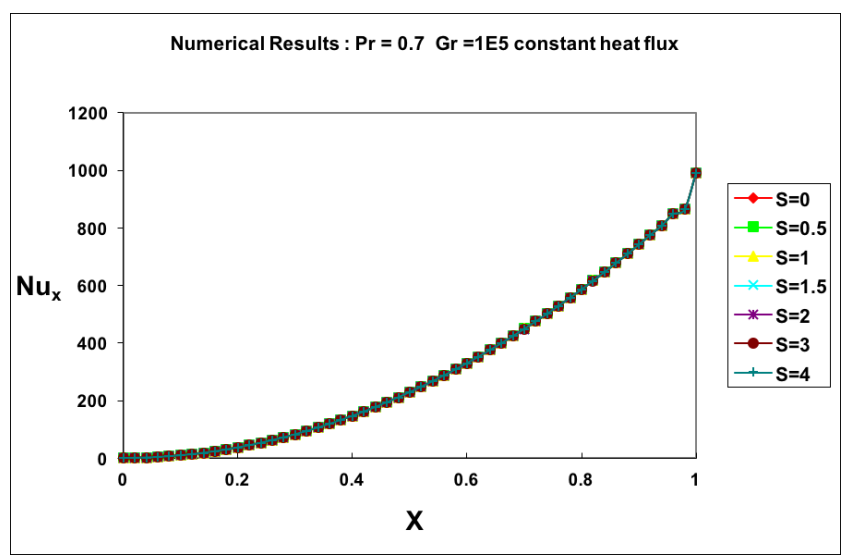

Fig. 12. Local Nusselt Number for $P r=0.7$.

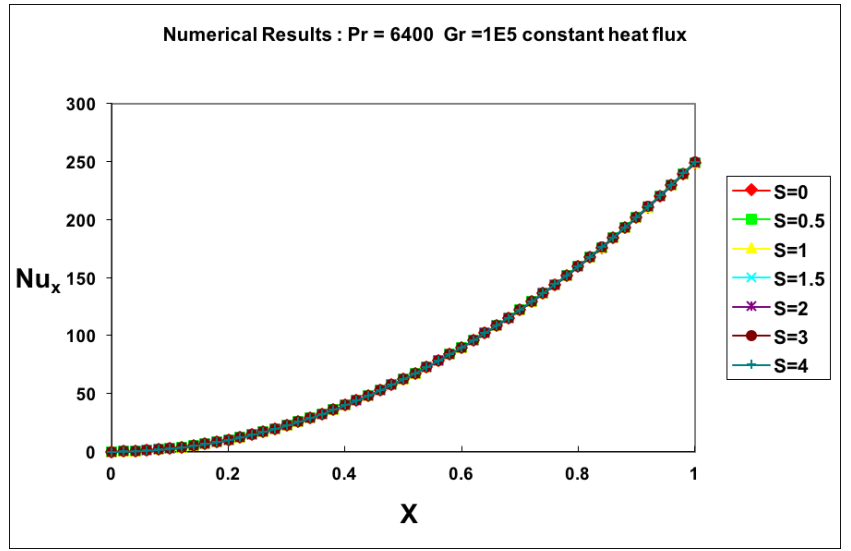

Fig. 13. Local Nusselt Number for $\operatorname{Pr}=6400$.

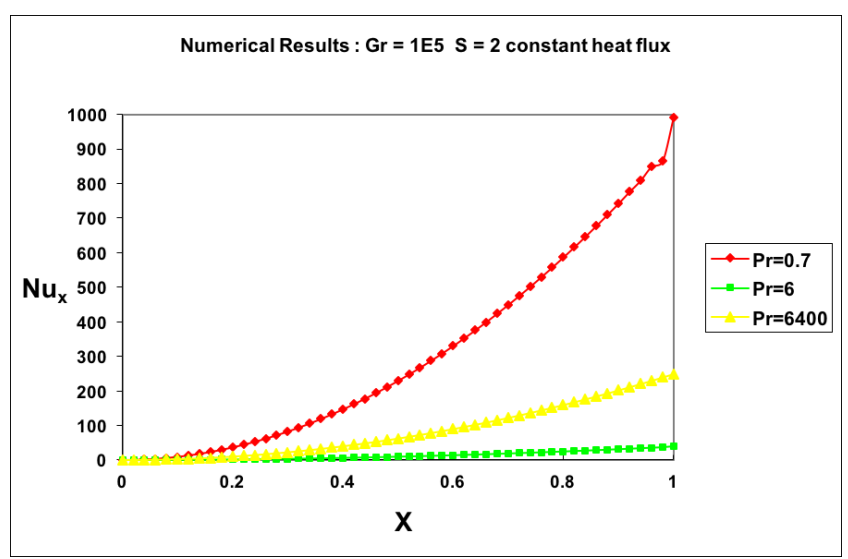

Fig. 14. Local Nusselt Number for $G r=1 \mathrm{E} 6$.

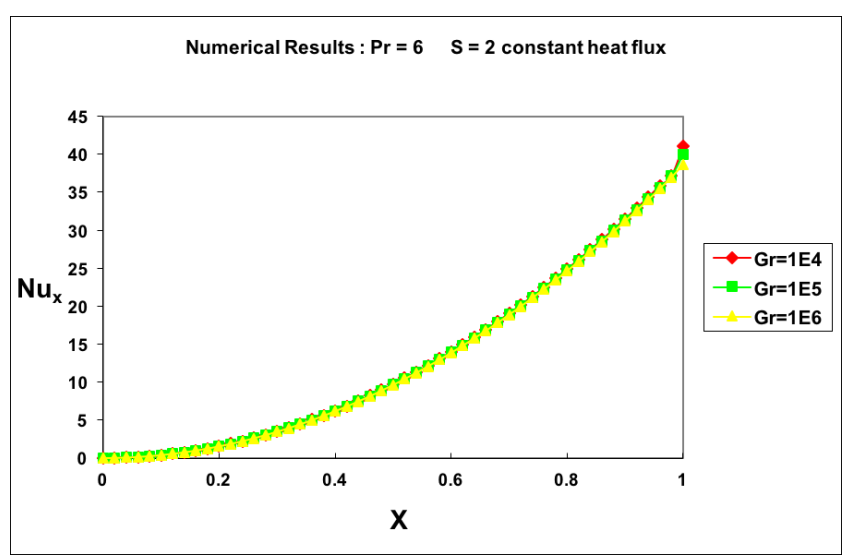

Fig. 15. Local Nusselt Number for $\operatorname{Pr}=6$. 


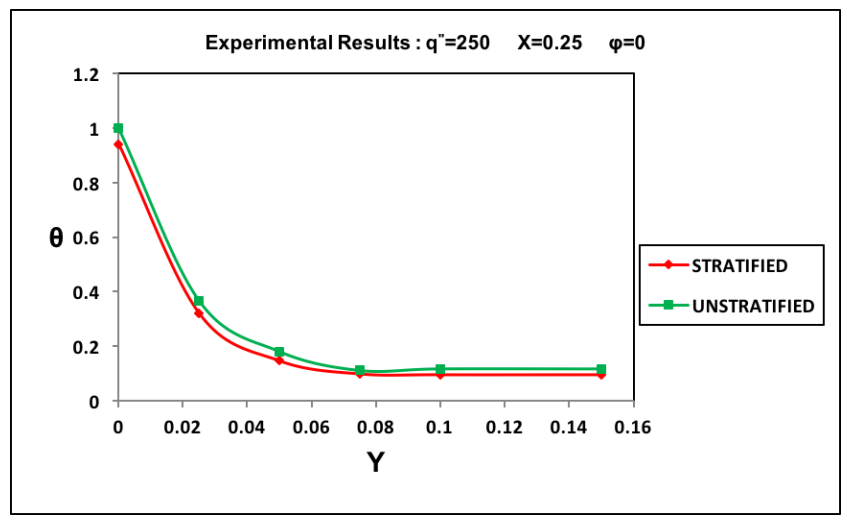

Fig. 16. Temperature profile at $X=0.25$.

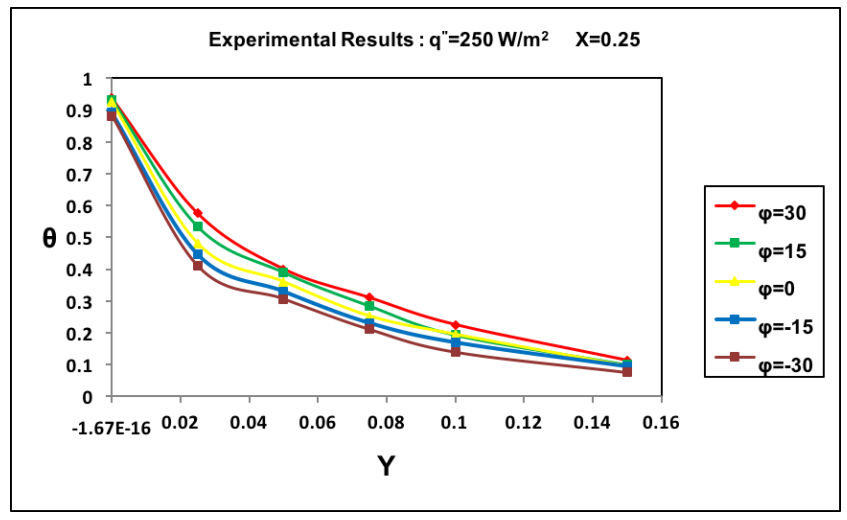

Fig. 17. Temperature profile based on the inclination angle at $X=0.25$.

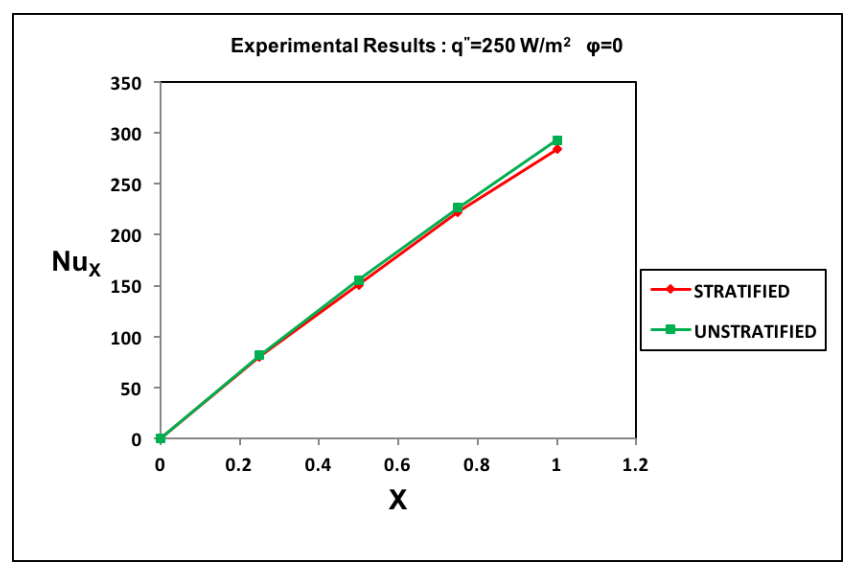

Fig. 18. Local Nusselt Number for stratified and unstratified medium.

\section{VERIFICATION}

The comparison between the experimental and theoretical temperature profile results for $\left(q "=250 \mathrm{~W} / \mathrm{m}^{2}\right.$ and $\left.\psi=0\right)$ is shown. The result reveals that the experimental temperature profile follows the same behavior as the present theoretical results but is approximately with means difference of $7.75 \%$. Thus, the comparison between the experimental and theoretical local Nusselt number results for $\mathrm{q} "=250 \mathrm{~W} / \mathrm{m}^{2}$ and $\psi=0$ reveals that the experimental local Nusselt number follows the same behavior as the present theoretical results but is approximately with mean difference of $20 \%$. The difference between the experimental and numerical results is due to inaccurate temperature measurement at the wall and flow blocking phenomenon occurring as result of horizontal rake. The deviations between the theoretical and experimental results may be due to the variation of Prandtl number in the ambient stratified fluid [14], and the thermal stratification is not perfectly linear in experimental investigation. Also, a comparison is made with the results achieved by previous studies. The effect of Prandlt number on temperature Profile agrees with results of [13]. Thermal boundary layer thickness agree with [14] (experimental study).

\section{CORRELATION OF AVERAGE HEAT TRANSFER DATA}

As a result of studying natural convection problems, the relation between average Nusselt numbers with Raleigh numbers and stratification parameters may be represented as a correlation relates these characteristics. The Multiple Regression method was used to find the correlation between them. These correlations for the range of Raleigh number from $0.7 \times 10^{4}$ to $6400 \times 10^{6}$, stratification parameter from 0 to 4 and heat flux $250 \mathrm{~W} / \mathrm{m}^{2}$. The correlation is presented as $N u_{\text {avg }}=130.6208-8.0577 \times 10^{-9} R a-1.272 S$.

\section{CONCLUSIONS}

Theoretical and experimental investigations of the natural convection heat transfer from a plane wall to a thermally stratified environment have been carried out in the present work to show the the significant effect of natural convection in a stratified media on the temperature profile, velocity profile and local Nusselt number. The results can be summarized as follows: 1.) For constant heat flux, Stratification parameter has the marginalized effect on the temperature, velocity profiles and local Nusselt number. 2.) The effect of stratification parameter is marginalized with the increase in Prandtl number. 3.) Thermal boundary layer was approximately independent on downstream coordinates. 4.) The reversal of temperature is strong at high Prandtl numbers and weaker at low numbers and the reversal of flow velocity is strong at low Prandtl numbers and weaker at high numbers. 5.) The increase in Grashof number does not practically vary the effect of stratification on temperature and velocity profiles. 6. ) As Prandtl number increases the Nusselt number first decrease, then increase. Comparison was made between present experimental and theoretical temperature profile and local Nusselt number. The temperature profile and local Nusselt number follows the same behavior with a mean difference of $7.75 \%$ for temperature profile and $20 \%$ for local Nusselt number. The present experimental and theoretical results also have been compared with the available previous studies for temperature profile, velocity profile and local Nusselt number, and give a good agreement.

An extension result of the present experimental and numerical works has been recommended for future work to study the natural convection heat transfer from a plane wall to a thermally stratified environment including: 1.)Studying the entropy generation during transient laminar natural convection in a stratified environment. 2.) Studying the considered parameters on the turbulent convection of the atmospheric boundary layer. 


\section{REFERENCES}

[1] T. E. Ahmed. Transient natural convection heat transfer from a plane wall to a thermally stratified media. $\mathrm{PhD}$ thesis, $\mathrm{M}$. Sc. Thesis, University of Technology, Baghdad, 2005.

[2] D. Angirasa and J. Srinivasan. Natural convection heat transfer from an isothermal vertical surface to a stable thermally stratified fluid. Journal of heat transfer, 114(4):917-923, 1992.

[3] R. Cheesewright. Natural convection from a plane, vertical surface in non-isothermal surroundings. International journal of Heat and Mass transfer, 10(12):1847-1859, 1967.

[4] C. C. Chen and R. Eichhorn. Natural convection from a vertical surface to a thermally stratified fluid. Journal of Heat Transfer, 98(3):446-451, 1976.

[5] R. K. Deka and B. C. Neog. Unsteady natural convection flow past an accelerated vertical plate in a thermally stratified fluid. Theoretical and Applied Mechanics, 36(4):261-274, 2009.

[6] J. P. Holman and W. J. Gajda. Experimental methods for engineers, volume 7. McGraw-Hill New York, 2001.

[7] I. Y. Hussain and N. K. Ali. Natural convection heat transfer from a plane wall to thermally stratified environment. $J$. of Engineering, (2):223-238, 2012.

[8] A. Ishak, R. Nazar, and I. Pop. Mixed convection boundary layer flow adjacent to a vertical surface embedded in a stable stratified medium. International Journal of Heat and Mass Transfer, 51(13):3693-3695, 2008.

[9] Y. Jaluria and K. Himasekhar. Buoyancy-induced two-dimensional vertical flows in a thermally stratified environment. Computers \& Fluids, 11(1):39-49, 1983.

[10] A. K. Kulkarni, H. R. Jacobs, and J. J. Hwang. Similarity solution for natural convection flow over an isothermal vertical wall immersed in thermally stratified medium. International journal of heat and mass transfer, 30(4):691-698, 1987.

[11] A. Pantokratoras. A note on the nusselt number adjacent to a vertical isothermal plate immersed in thermally stratified water at low temperatures. International journal of heat and fluid flow, 24(2):278-281, 2003.

[12] S. C. Saha and M. A. Hossain. Natural convection flow with combined buoyancy effects due to thermal and mass diffusion in a thermally stratified media. Nonlinear Analysis, 9(1):89-102, 2004.

[13] G. Singh, P. R. Sharma, and A. J. Chamkha. Effect of thermally stratified ambient fluid on mhd convective flow along a moving non-isothermal vertical plate. International Journal of Physical Sciences, 5(3):208-215, 2010.

[14] J. Tanny and J. Cohen. The mean temperature field of a buoyancyinduced boundary layer adjacent to a vertical plate immersed in a stratified medium. International journal of heat and mass transfer, 41(14):2125-2130, 1998.

[15] K. T. Yang, J. L. Novotny, and Y. S. Cheng. Laminar free convection from a nonisothermal plate immersed in a temperature stratified medium. International journal of Heat and Mass transfer, 15(5):1097-1109, 1972. 